\title{
Suitability of capillary blood obtained by a minimally invasive lancet technique to detect subclinical ketosis in dairy cows by using 3 different electronic hand-held devices
}

\author{
P. Kanz, ${ }^{*}$ M. Drillich, ${ }^{*}$ D. Klein-Jöbstl, ${ }^{*}$ B. Mair, ${ }^{*}$ S. Borchardt, $\dagger^{1}$ L. Meyer, $\dagger$ I. Schwendenwein, $\ddagger$ \\ and M. Iwersen*2 \\ ${ }^{*}$ Clinical Unit for Herd Health Management in Ruminants, University Clinic for Ruminants, Department for Farm Animals \\ and Veterinary Public Health, University of Veterinary Medicine Vienna, 1210 Vienna, Austria \\ †FirstFarms Slovakia, 90068 Plavecký Štvrtok, Slovakia \\ $\ddagger$ Central Clinical Pathology Unit, Department for Pathobiology, University of Veterinary Medicine Vienna, 1210 Vienna, Austria
}

\begin{abstract}
The objective of this study was to evaluate the suitability of capillary blood obtained by a minimally invasive lancet technique to detect subclinical ketosis in 49 prepartum and 191 postpartum Holstein-Friesian cows using 3 different electronic hand-held devices [FreeStyle Precision (FSP, Abbott), GlucoMen LX Plus (GLX, A. Menarini), NovaVet (NOV, Nova Biomedical)]. The $\beta$-hydroxybutyrate (BHBA) concentration in serum harvested from coccygeal blood samples was analyzed in a laboratory and used as a reference value. Capillary samples were obtained from the skin of the exterior vulva by using 1 of 3 different lancets. In all samples, the concentration of BHBA was immediately analyzed with all 3 hand-held devices used in random order. All lancets used in the study were eligible for capillary blood collection but differed in the total number of incisions needed. Spearman correlation coefficients between the BHBA concentrations in capillary blood and the reference test were highly significant with $83 \%$ for the FSP, $73 \%$ for the NOV, and $63 \%$ for the GLX. Using capillary blood, the FSP overestimated the mean BHBA concentration compared with the reference test $(+0.08 \mathrm{mmol} / \mathrm{L})$, whereas the GLX and NOV underestimated the mean concentration $(-0.07$ and $-0.01 \mathrm{mmol} / \mathrm{L}$ ). When a BHBA concentration of 1.2 $\mathrm{mmol} / \mathrm{L}$ in serum was used to define subclinical ketosis, the corresponding analyses of receiver operating characteristics resulted in optimized thresholds for capillary blood of $1.1 \mathrm{mmol} / \mathrm{L}$ for the NOV and GLX devices, and of $1.0 \mathrm{mmol} / \mathrm{L}$ for the FSP. Based on these thresholds, sensitivities (Se) and specificities (Sp) were 89 and $84 \%$
\end{abstract}

\footnotetext{
Received October 10, 2014.

Accepted May 12, 2015.

${ }^{1}$ Current address: Clinic for Animal Reproduction, Faculty of Veterinary Medicine, Freie Universität Berlin, Berlin, Germany.

${ }^{2}$ Corresponding author: Michael.Iwersen@vetmeduni.ac.at
}

for the NOV, 80 and $89 \%$ for the GLX, and 100 and $76 \%$ for the FSP. Based on a serum BHBA concentration of $1.4 \mathrm{mmol} / \mathrm{L}$, analyses of receiver operating characteristics resulted in optimized cut-offs of $1.4 \mathrm{mmol} / \mathrm{L}$ for the FSP (Se 100\%, Sp 92\%), $1.3 \mathrm{mmol} / \mathrm{L}$ for the NOV (Se 80\%, Sp 95\%), and $1.1 \mathrm{mmol} / \mathrm{L}$ (Se 90\%, Sp $85 \%$ ) for the GLX. Using these optimized thresholds for the specific hand-held meters, no significant differences between the devices in Se and Sp to detect subclinical ketosis in coccygeal blood were observed. Calculated test characteristics for analyzing capillary blood using the hand-held devices were numerically smaller compared with blood obtained from a coccygeal vessel, but overlapping confidence intervals indicate no statistical difference between the origin of the sample. Hence, this procedure seems to be suitable for ketosis monitoring in dairy cows, but further validation with more data from different farms is recommended.

Key words: cow, ketosis, capillary blood, $\beta$-hydroxybutyrate, diagnostic test

\section{INTRODUCTION}

Subclinical ketosis (SCK) is defined as a metabolic disorder with an increased ketone body concentration in the absence of clinical symptoms of ketosis (Andersson, 1988; Duffield et al., 1998; Rollin et al., 2010). Commonly used thresholds to define SCK are BHBA concentrations in blood of 1.2 and $1.4 \mathrm{mmol} / \mathrm{L}$ (Geishauser et al., 1998; Duffield et al., 2009). At herd level, a target incidence of SCK below 20\% (using a cut-off of the BHBA concentration in serum of 1.4 mmol/L) was recommended by Cook et al. (2006).

The occurrence of SCK in dairy herds in the periparturient period, caused by a negative energy balance, represents an important challenge for dairy farmers. Several studies have shown that SCK is associated with an increased risk for the occurrence of secondary diseases such as clinical ketosis, displaced abomasum, me- 
tritis, mastitis, and lameness (Geishauser et al., 1998; Duffield et al., 2009; Suthar et al., 2013). Additionally, decreased milk yield (Dohoo and Martin, 1984; Duffield et al., 1997) and impaired reproductive performance (Walsh et al., 2007a,b; Chapinal et al., 2012) are associated with the occurrence of SCK. Based on BHBA concentrations in blood of $\geq 1.2 \mathrm{mmol} / \mathrm{L}$, Suthar et al. (2013) reported an overall prevalence of SCK for 10 European countries of $21.8 \%$, ranging from 11.2 to $36.6 \%$, within 2 wk after calving. Reported prevalence for North American dairy herds ranged from 8.9 to $43.2 \%$ within the first 2 mo of lactation (Dohoo and Martin, 1984; Geishauser et al., 1998; McArt et al., 2012). Recently, an increased BHBA concentration before calving has been associated with a detrimental effect on milk yield and animal health (Chapinal et al., 2011, 2012). Animals showing a BHBA concentration in serum $\geq 0.7 \mathrm{mmol} / \mathrm{L}$ within the last week of gestation were at higher risk of early culling (Roberts et al., 2012).

Considering the abovementioned aspects, monitoring of dairy herds for SCK is reported to be an appropriate measure for disease prevention and improvement of stock management efficiency in dairy farming (Cook et al., 2006). In several studies, the determination of BHBA concentrations in serum or plasma with standard laboratory methods was defined as a reference test for diagnosing of SCK (Duffield et al., 1998; Geishauser et al., 2000). This method, however, is inconvenient for a broader surveillance because of its costs in terms of time and money. The possible delay in treatment of animals suffering from SCK, because of shipping and analyzing a blood sample at an external laboratory, might have a negative effect on animal welfare as well. Routine testing of animals at risk for SCK by the farmer can be used on a cow level (to guide individual treatment) as well as on a herd level (to evaluate transition management and feeding).

Within the last 2 decades, several point-of-care tests have been developed and were evaluated for dairy cows to detect ketones in urine (Carrier et al., 2004; Krogh et al., 2011), milk (Geishauser et al., 1998, 2000; Carrier et al., 2004; Krogh et al., 2011), and whole blood (Iwersen et al., 2009, 2013; Mahrt et al., 2014b). Cowside ketone tests for blood may be preferred because they are most close to the reference test for SCK based on BHBA concentrations in serum or plasma (Geishauser et al., 1998, 2000; Carrier et al., 2004; Iwersen et al., 2009, 2013). Only few studies, however, performed a direct comparison between different hand-held meters for detection of SCK (Iwersen et al., 2013).

To our knowledge, only venous or arterial blood samples or both have been evaluated for monitoring of ketosis using electronic hand-held devices. A dis- advantage of this testing method is its more invasive sampling technique compared with milk- and urinebased systems. Additionally, in many countries (e.g., Germany, Switzerland, and the Netherlands), national legislation prohibits conventional blood sampling by laypersons (e.g., farmers). Capillary blood might be an alternative, as sampling is considered less invasive and easier to achieve compared with the conventional blood-sampling procedures. The permission of obtaining capillary blood by the farmer using a minimally invasive technique for diagnostic purposes is already in consideration by the authorities in Austria, for instance.

The primary objective of this study was to test whether capillary blood obtained from the skin of the exterior vulva by using a minimally invasive lancet technique is suitable to detect SCK in pre- and postpartum dairy cows. Secondary objectives were to test 3 different lancets for obtaining the capillary blood and to test 3 commercially available hand-held devices within the same experiment. The BHBA concentrations in coccygeal blood were analyzed in a laboratory to be used as reference value and assessed with the hand-held devices to distinguish between the effect of the device and the type of blood used for the cow-side testing.

\section{MATERIALS AND METHODS}

\section{Experimental Design}

The study was approved by the institutional ethics committee of the University of Veterinary Medicine, Vienna, and the national authority according to $\S 26$ of the Law for Animal Experiments, Tierversuchsgesetz 2012 - TVG 2012 (GZ 68.205/0007-II/3b/2014) as well as by the Slovakian Regional Veterinary Food Administration (428/2014). The study was conducted on 4 sampling dates between March and April 2014 on a Slovakian dairy farm, keeping approximately 2,700 Holstein-Friesian cows and additional youngstock. Cows were housed in freestall barns with high bed cubicles. Rubber mats with dried slurry separator material were used as cubicle bedding. The average ECM yield (based on $4.0 \%$ butterfat and $3.4 \%$ protein) was $9,165 \mathrm{~kg}$ in 2013.

In total, 240 primi- and multiparous cows predominantly within the transition period were enrolled in this study. Each cow was only tested at one sampling date within the study period. At each of the 4 farm visits, samples of approximately 50 randomly selected animals in the fresh cow pen and of approximately 12 randomly selected cows in the close-up pen were taken.

The final data set used for the statistical analyses consisted of $34(14.2 \%)$ animals in first lactation, 97 (40.4\%) in second lactation, and 109 (45.4\%) in third 
or greater lactation. Considering the 240 enrolled animals, $49(20.4 \%)$ were tested between $21 \mathrm{~d}$ and $12 \mathrm{~h}$ prepartum (median: $4 \mathrm{~d}$ prepartum) and 191 (79.6\%) animals were sampled between $1 \mathrm{~d}$ and $29 \mathrm{~d}$ postpartum (median: $8 \mathrm{~d}$ postpartum).

Three electronic hand-held devices [FreeStyle Precision (FSP, Abbott GmbH \& Co. KG, Wiesbaden, Germany), GlucoMen LX Plus (GLX, A. Menarini GmbH, Vienna, Austria), NovaVet (NOV, Nova Biomedical, Waltham, MA)] were used to analyze the BHBA concentration in capillary blood as well as in a whole-blood sample obtained from a coccygeal vessel. To obtain capillary blood, 3 different types of disposable lancets [Microtainer Contact-Activated Lancet (MT, Becton Dickinson, Franklin Lakes, NJ), SafetyLancets special (SL, Med Trust Handelsgesm.b.H., Marz, Austria), MiniCollect Safety Lancets (MC, Greiner Bio-One International AG, Kremsmünster, Austria)] were used. For sampling procedures, the skin of the exterior vulva was cleaned with a paper towel, disinfected, and then punctured using a minimally invasive lancet to obtain a single blood drop. The penetration depth was $2 \mathrm{~mm}$ for all types of lancets, with blade widths differing between $0.8 \mathrm{~mm}(\mathrm{SL})$ and $1.5 \mathrm{~mm}$ (MT and MC). If the obtained blood volume was insufficient for an accurate measurement with all 3 electronic hand-held devices, the bleeding was enforced by softly squeezing the skin of the exterior vulva. If this still was unsuccessful, another puncture approximately $1 \mathrm{~cm}$ lateral from the first incision was performed.

The animal identification number, the date of sampling, the number of required punctures for each specific lancet, and the analyzed results of all 3 hand-held devices for capillary and coccygeal blood were recorded onto a data-capture form. The type of lancet that had to be used was preassigned on this data-capture form, generated by using the random function in Excel (version 6.1.760, Microsoft Corporation, Redmond, WA).

The BHBA concentration of the capillary blood drop was immediately analyzed using all 3 electronic devices in random order. After inserting the test strips into the hand-held devices, the front edges of the strips were dipped directly onto the drop of blood. The operating principle was similar for all 3 devices and has been described elsewhere (Iwersen et al., 2009). The amount of blood required for analyses ranges between $0.8 \mu \mathrm{L}$ (GLX, NOV) and $1.5 \mu \mathrm{L}$ (FSP). The coccygeal blood samples were obtained with vacuum tubes coated with a clot activator for serum collection (Vacuette, $9 \mathrm{~mL}$, Greiner Bio-One $\mathrm{GmbH}$ ). The samples were immediately tested with all 3 electronic devices in random order, too, by dipping the sensor of the strips onto the surface of the blood-filled tube. All sampling procedures per animal were completed within 5 min. After clotting, the serum tubes were centrifuged $\left(10 \mathrm{~min}, 18^{\circ} \mathrm{C}, 2,200\right.$ $\times g$ ), and serum was divided into 2 aliquots and stored at a temperature of $-18^{\circ} \mathrm{C}$ until further analysis at the laboratory of the Central Clinical Pathology Unit (CCPU), University of Veterinary Medicine, Vienna, Austria. The concentration of BHBA in serum was analyzed in the laboratory using a colorimetric enzymatic reaction (Ranbut D-3-hydroxybutyrate; Randox Laboratories Ltd., Antrim, UK) with an automated wet chemistry analyzer (Cobas 6000/501c; Roche Diagnostics International AG, Rotkreuz, Switzerland) as described by Pichler et al. (2014). The determination of BHBA concentrations in serum at the CCPU was defined as the reference test in our study.

To evaluate the intraassay variability of the laboratory analyses, a subset of 20 aliquots of blood samples, taken from one cow, was randomly placed among the samples obtained from the study animals. The mean \pm standard deviation (SD) BHBA concentration of those samples analyzed with the wet chemistry analyzer at the CCPU was $1.19 \pm 0.01 \mathrm{mmol} / \mathrm{L}$, resulting in a coefficient of variation of $1.13 \%$.

Intra- and interassay coefficients of variations $(\mathbf{C V})$ were furthermore calculated for each type of handheld devices. For this, 3 blood samples with different BHBA concentrations based on FSP measurements with low $(0.3 \mathrm{mmol} / \mathrm{L})$, medium $(1.3 \mathrm{mmol} / \mathrm{L})$, and high $(2.1 \mathrm{mmol} / \mathrm{L})$ BHBA concentrations were tested 10 times with one device (intraassay) and additionally with 10 different devices of the same type (interassay). Analyzing low BHBA concentrations resulted in intraassay and interassay CV of $>10 \%$ for all 3 devices, ranging from 12.4 to $19.7 \%$. Analyzed medium and high BHBA concentrations revealed CV less than $10 \%$ for all devices, except interassay CV of $11.5 \%$ for medium BHBA concentrations determined by NOV. Average intraassay CV ranged between $7.9 \%$ (FSP) and 10.9\% (NOV); average interassay $\mathrm{CV}$ ranged between $7.2 \%$ (FSP) and $13.1 \%$ (NOV).

\section{Statistical Analyses}

For statistical analyses SPSS Statistics for Windows (version 20.0; IBM Deutschland GmbH, Ehningen, Germany), MedCalc for Windows (version 12.4; MedCalc Software, Ostend, Belgium), and BiAS for Windows (version 10.06; Epsilon-Verlag, Darmstadt, Germany) were used. The level of significance for all statistical tests was set at $P=0.05$. To compare the results of our study with others, traditional measures, Kendall's tau $(\tau)$ and Spearman's rho $(\rho)$ correlation coefficients, were calculated for the BHBA concentrations analyzed in capillary or coccygeal blood for each hand-held device and their corresponding BHBA concentration 
determined at the CCPU. A correlation coefficient describes a statistical relationship between data but does not prove any agreement between the compared results and does not detect any constant or proportional difference between 2 methods (Bilić-Zulle, 2011). Hence, the agreement between the results of each hand-held meter and the reference test was evaluated using the method described by Bland and Altman (1986).

Furthermore, regression analyses as recommended by Passing and Bablok (1983) were conducted to compare the BHBA concentration, analyzed in capillary and coccygeal blood with all 3 electronic hand-held devices (i.e., as predictor in the model), with the laboratory results (i.e., the specific outcome variable in the model). By performing the Passing-Bablok regression, the outcome does not depend upon the assignment of the methods to $x$ and $y$. Confidence intervals for the intercept $(a)$ as a measure of the systematic error between both methods and the slope $(b)$ of the regression line, reflecting the proportional differences between the 2 methods, are calculated. No specific assumptions regarding the distributions of the expected values or error terms are required for the abovementioned regression analyses, but for a meaningful estimation of $a$ and $b$, a linear relationship as well as a high correlation between the data measured by 2 analytical methods is required (Passing and Bablok, 1983). The assumption of linearity of the data was tested by the cumulative sum linearity (Cusum) test.

Analyses of receiver operating characteristics (ROC) were performed on BHBA concentrations to calculate best thresholds to detect SCK (based on BHBA concentrations of 1.2 and $1.4 \mathrm{mmol} / \mathrm{L}$ in serum analyzed with the reference test) in capillary and coccygeal blood for each hand-held meter, and sensitivities (Se) as well as specificities (Sp) were reported for all devices using these optimized thresholds. Best thresholds analyses were based on maximizing the Youden index $(J=\mathrm{Se}+\mathrm{Sp}-1)$. The area under the ROC curve (AUC-ROC) represents how well a parameter can distinguish between 2 diagnostic groups; it is a measure for the overall discriminative capacity of the test. An AUC-ROC of $100 \%$ represents a perfect test; an AUCROC of $50 \%$ represents a worthless test (Swets, 1988). The numbers of incisions needed using the 3 different lancets to obtain capillary blood were compared by applying a Fisher's exact test.

\section{RESULTS}

The BHBA concentrations in serum exceeded the threshold of $0.7 \mathrm{mmol} / \mathrm{L}$ in $34.7 \%(\mathrm{n}=17)$ of the cows that were sampled prepartum $(\mathrm{n}=49$; median BHBA concentration: $0.66 \mathrm{mmol} / \mathrm{L}$, minimum: $0.35 \mathrm{mmol} / \mathrm{L}$, maximum: $1.75 \mathrm{mmol} / \mathrm{L})$. Considering the samples taken postpartum $(\mathrm{n}=191$; median BHBA concentration: $0.66 \mathrm{mmol} / \mathrm{L}$, minimum: $0.35 \mathrm{mmol} / \mathrm{L}$, maximum: 1.75 $\mathrm{mmol} / \mathrm{L}$ ), the BHBA concentration in serum exceeded the threshold of $1.2 \mathrm{mmol} / \mathrm{L}$ in $16.2 \%(\mathrm{n}=31)$ and the threshold of $1.4 \mathrm{mmol} / \mathrm{L}$ in $8.9 \%(\mathrm{n}=17)$. Analyzing only animals within the first 2 wk of lactation $(\mathrm{n}=$ 149), prevalence of SCK remained at similar levels with 14.8 and $8.1 \%$, respectively.

All lancets used in the study were eligible for capillary blood collection but differed in the total number of incisions needed $(P=0.047)$. Capillary blood could be obtained with first incision in $85 \%(\mathrm{n}=68)$ using the SL, 95\% ( $\mathrm{n}=76)$ using the MT, and 96\% ( $\mathrm{n}=$ 77) using the MC lancet. An additional second (and third) incision had to be performed for the SL in 9 (3) cases, using the MT in 4 (0) and the MC in 2 (1) cases. No discomforting reactions of the animals could be observed related to the incision of the lancets.

In total, 480 blood samples (240 capillary and coccygeal samples, each) were analyzed with the hand-held devices. The Spearman's correlation coefficient $\rho$ (and Kendall's $\tau$ ) between the reference test and the BHBA concentrations analyzed in coccygeal blood using the hand-held devices were $0.95(0.85)$ for the FSP, 0.85 $(0.70)$ for the NOV, and 0.81 (0.67) for the GLX device $(P<0.01$ for all devices and both parameter $)$. Comparing the hand-held results of capillary blood with the reference test yielded in $\rho$ (and $\tau$ ) of $0.83(0.67)$ for the FSP, $0.73(0.58)$ for the NOV, and $0.62(0.48)$ for the GLX device $(P<0.01$ for all devices and both parameters). Further descriptive statistical parameters for the BHBA concentrations analyzed in coccygeal and capillary blood using the hand-held devices as well as the laboratory results are presented in Table 1. As shown in Figure 1, the median BHBA concentration of the samples was always smaller in coccygeal blood than in capillary blood, no matter which hand-held device was used.

As presented in the Bland-Altman plots (Figure 2) using coccygeal blood, the mean \pm SD BHBA concentration analyzed with the reference test was overestimated (positive bias, $P<0.01$ ) by $0.02 \pm 0.12 \mathrm{mmol} / \mathrm{L}$ using the FSP, whereas NOV and GLX underestimated $(P<$ $0.05)$ the mean $\pm \mathrm{SD}$ BHBA concentration (negative bias) by $0.06 \pm 0.17$ and $0.10 \pm 0.21 \mathrm{mmol} / \mathrm{L}$, respectively. Using capillary blood, the FSP overestimated ( $P$ $<0.01$ ) the mean $\pm \mathrm{SD}$ BHBA concentration compared with the reference test by $0.08 \pm 0.19$, whereas NOV and GLX underestimated $(P<0.01)$ the mean \pm SD BHBA concentration by $0.01 \pm 0.43$ and $0.07 \pm 0.36$, respectively.

Except for measurements in coccygeal blood using the NOV, the Cusum test detected no significant devia- 
Table 1. Descriptive statistics of the BHBA concentration analyzed in coccygeal and capillary blood of 49 prepartum $^{1}$ and 191 postpartum ${ }^{2}$ Holstein-Friesian cows predominately taken between 2 wk before and 4 wk after calving using 3 different hand-held devices as well as in serum analyzed at the laboratory

\begin{tabular}{|c|c|c|c|c|c|c|c|}
\hline \multirow[b]{2}{*}{ Parameter } & \multirow{2}{*}{$\begin{array}{l}\text { Serum, }{ }^{3} \\
\text { laboratory }\end{array}$} & \multicolumn{3}{|c|}{ Capillary blood ${ }^{4}$} & \multicolumn{3}{|c|}{ Coccygeal blood } \\
\hline & & FSP & GLX & $\mathrm{NOV}$ & FSP & GLX & $\mathrm{NOV}$ \\
\hline Number of samples & 240 & 240 & 240 & 240 & 240 & 240 & 240 \\
\hline Mean $(\mathrm{mmol} / \mathrm{L})$ & 0.89 & 0.97 & 0.81 & 0.87 & 0.91 & 0.80 & 0.83 \\
\hline $\mathrm{SD}(\mathrm{mmol} / \mathrm{L})$ & 0.44 & 0.47 & 0.42 & 0.41 & 0.41 & 0.43 & 0.39 \\
\hline Median (mmol/L) & 0.77 & 0.80 & 0.80 & 0.80 & 0.80 & 0.70 & 0.80 \\
\hline Interquartile range $(\mathrm{mmol} / \mathrm{L})$ & 0.37 & 0.40 & 0.40 & 0.50 & 0.50 & 0.50 & 0.40 \\
\hline
\end{tabular}

${ }^{1}$ Median: $4 \mathrm{~d}$, minimum: $21 \mathrm{~d}$, maximum: $12 \mathrm{~h}$ prepartum.

${ }^{2}$ Median: $8 \mathrm{~d}$, minimum: $1 \mathrm{~d}$, maximum: $29 \mathrm{~d}$ postpartum.

${ }^{3}$ Obtained from coccygeal blood (reference test).

${ }^{4}$ FSP $=$ FreeStyle Precision (Abbott GmbH and Co. KG, Wiesbaden, Germany); GLX = GlucoMen LX Plus

(A. Menarini GmbH, Vienna, Austria); NOV = NovaVet (Nova Biomedical, Waltham, MA).

tion from linearity between the results of the reference test and the measurements of the hand-held devices. For measurements in capillary and coccygeal blood,
Passing-Bablok regression detected significant proportional and systematic differences for the FSP and GLX devices compared with the reference method (Table

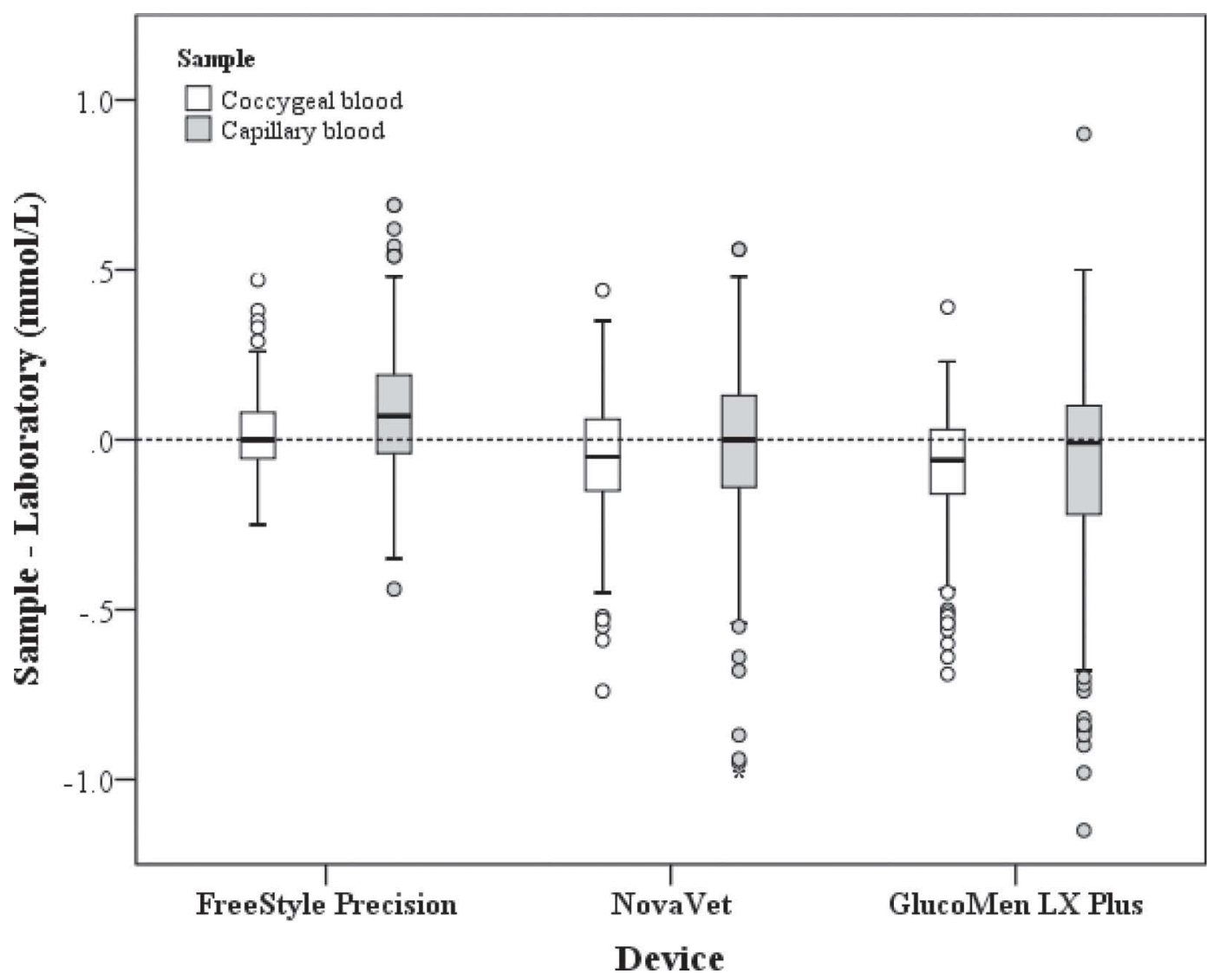

Figure 1. Differences between the BHBA concentrations in serum obtained from 49 prepartum and 191 postpartum Holstein-Friesian cows predominately taken between 2 wk before (median: $4 \mathrm{~d}$, minimum: $21 \mathrm{~d}$, maximum: $12 \mathrm{~h}$ prepartum) and 4 wk after calving (median: 8 d, minimum: $1 \mathrm{~d}$, maximum: $29 \mathrm{~d}$ postpartum) analyzed at the laboratory (reference test) and the concentrations measured with 3 different hand-held devices either in whole blood obtained from a tail vessel or in capillary blood. The heavy black line inside each box marks the median (50th percentile); lower and upper hinges mark the 25th and 75th percentiles. Whiskers end at the smallest and largest statistical values that are not outliers; outliers and extreme values are designated by $\bigcirc$ and *. FreeStyle Precision (Abbott GmbH and Co. KG, Wiesbaden, Germany); NovaVet (Nova Biomedical, Waltham, MA); GlucoMen LX Plus (A. Menarini GmbH, Vienna, Austria). 

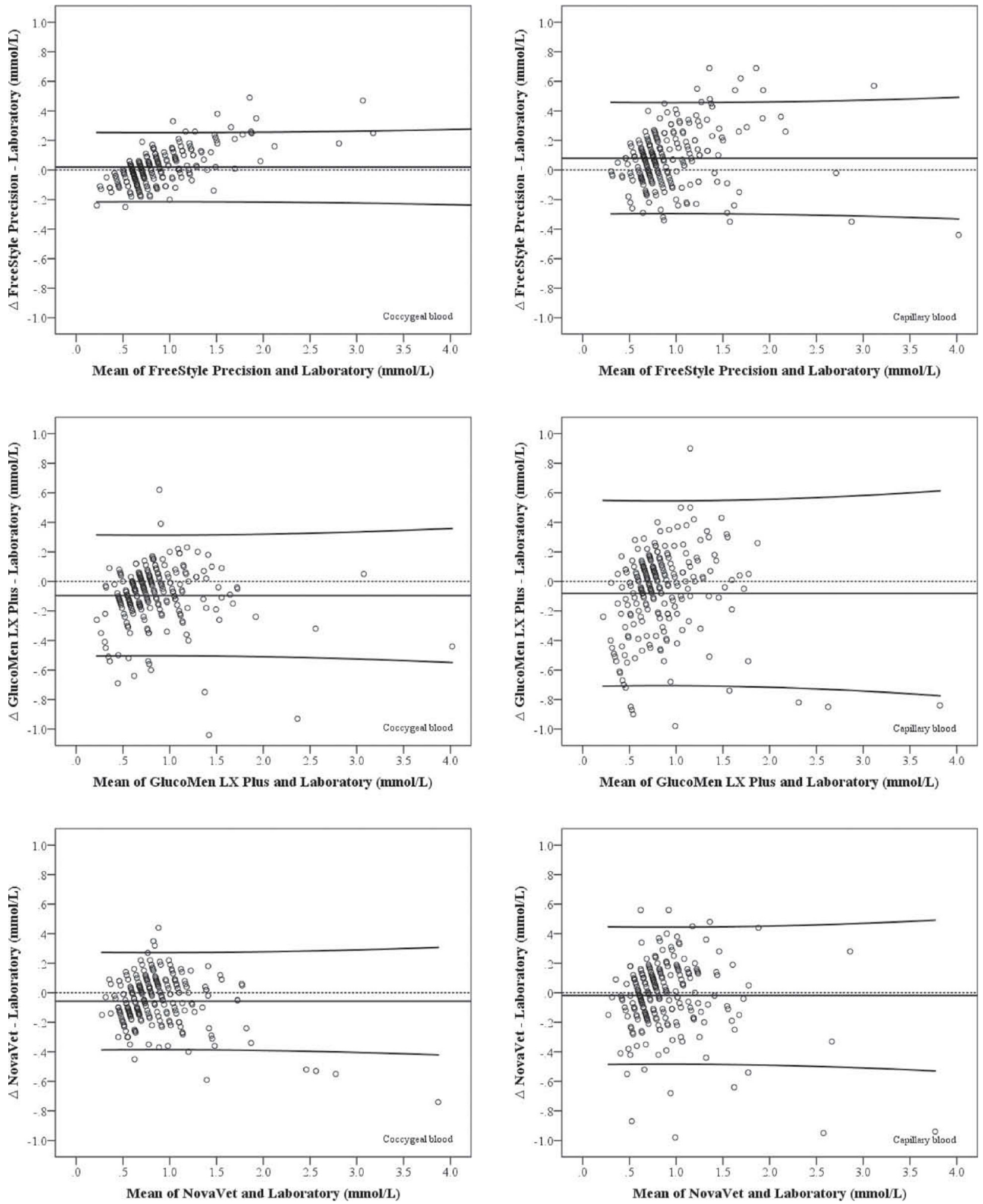

Figure 2. Differences in BHBA concentrations measured with 3 different electronic hand-held devices in coccygeal (left) or capillary (right) blood and the reference test against their mean. Blood samples were obtained from 49 prepartum and 191 postpartum Holstein-Friesian cows and were predominately taken between 2 wk before (median: $4 \mathrm{~d}$, minimum: $21 \mathrm{~d}$, maximum: $12 \mathrm{~h}$ prepartum) and 4 wk after calving (median: $8 \mathrm{~d}$, minimum: $1 \mathrm{~d}$, maximum: $29 \mathrm{~d}$ postpartum). The solid line in the middle represents the mean; the solid upper and lower lines represent the mean \pm 2 SD. FreeStyle Precision (Abbott GmbH and Co. KG, Wiesbaden, Germany); GlucoMen LX Plus (A. Menarini GmbH, Vienna, Austria); NovaVet (Nova Biomedical, Waltham, MA). 
Table 2. Differences between the BHBA concentrations analyzed by 3 different hand-held devices ${ }^{1}$ and the laboratory results using the Bland-Altman analysis method and Passing-Bablok regression analysis in samples obtained from 49 prepartum $^{2}$ and 191 postpartum $^{3}$ Holstein-Friesian cows predominately taken between 2 wk before and 4 wk after calving

\begin{tabular}{|c|c|c|c|}
\hline Item & FSP & GLX & NOV \\
\hline \multicolumn{4}{|l|}{ Capillary blood } \\
\hline \multicolumn{4}{|l|}{ Passing-Bablok } \\
\hline Slope $(b)$ & 1.19 & 1.25 & 1.11 \\
\hline $\mathrm{CI}_{95}{ }^{4}$ for $b$ & 1.11 to 1.27 & 1.11 to 1.42 & 1.00 to 1.25 \\
\hline Intercept $(a)$ & -0.07 & -0.21 & -0.08 \\
\hline $\mathrm{CI}_{95}$ for $a$ & -0.14 to -0.01 & -0.37 to -0.11 & -0.18 to 0.00 \\
\hline \multicolumn{4}{|l|}{ Bland-Altman } \\
\hline $\operatorname{Bias}(d)$ & 0.08 & -0.07 & -0.01 \\
\hline $\mathrm{SD}$ of $d$ & 0.19 & 0.36 & 0.43 \\
\hline \multicolumn{4}{|l|}{ Coccygeal blood } \\
\hline \multicolumn{4}{|l|}{ Passing-Bablok } \\
\hline$b$ & 1.22 & 1.11 & 1.05 \\
\hline $\mathrm{CI}_{95}$ for $b$ & 1.18 to 1.25 & 1.03 to 1.19 & 0.98 to 1.13 \\
\hline$a$ & -0.16 & -0.16 & -0.09 \\
\hline $\mathrm{CI}_{95}$ for $a$ & -0.18 to -0.12 & -0.22 to -0.08 & -0.16 to -0.03 \\
\hline \multicolumn{4}{|l|}{ Bland-Altman } \\
\hline$d$ & 0.02 & -0.10 & -0.06 \\
\hline SD of $d$ & 0.12 & 0.21 & 0.17 \\
\hline
\end{tabular}

${ }^{1} \mathrm{FSP}=$ FreeStyle Precision (Abbott GmbH and Co. KG, Wiesbaden, Germany); GLX = GlucoMen LX Plus (A. Menarini GmbH, Vienna, Austria); NOV = NovaVet (Nova Biomedical, Waltham, MA).

${ }^{2}$ Median: $4 \mathrm{~d}$, minimum: $21 \mathrm{~d}$, maximum: $12 \mathrm{~h}$ prepartum.

${ }^{3}$ Median: 8 d, minimum: $1 \mathrm{~d}$, maximum: $29 \mathrm{~d}$ postpartum.

${ }^{4} \mathrm{CI}_{95}=95 \%$ CI.

2). The results for the NOV for capillary blood were comparable with the reference test.

Analyses of ROC were performed using threshold BHBA concentrations (analyzed at the CCPU) of 1.2 and $1.4 \mathrm{mmol} / \mathrm{L}$ to define SCK to assess the best possible test characteristics for the various cow-side test options evaluated in this study. For GLX and NOV all optimized thresholds were lower compared with the reference value, whereas for the FSP, 3 of 4 evaluated thresholds were equal and 1 was lower (Table 3 ).

Except for the GLX used with capillary blood, overall accuracies of the hand-held devices were excellent (AUC-ROC $\geq 90 \%$ ) for diagnosing SCK in capillary and coccygeal blood, compared with the reference test defining SCK at a BHBA concentration of 1.2 and $1.4 \mathrm{mmol} / \mathrm{L}$ in serum, respectively. Evaluated accuracies for the GLX used with capillary blood were classified as good (AUC-ROC between 80 and <90\%). Sensitivities ranged between 80 and $100 \%$ for capillary blood and between 85 and 100\% for coccygeal blood. Corresponding Sp ranged between 76 and $95 \%$ with capillary blood and between 83 and $98 \%$ when coccygeal blood was used. Results for the AUC-ROC were in all cases highly significantly different from $50 \%$ (area under the diagonal line, indicating that a test does not have the ability to distinguish between 2 diagnostic groups) and ranged between $87 \%$ (GLX) and 100\% (FSP).

\section{DISCUSSION}

To our knowledge, this is the first study, evaluating the use of capillary blood for diagnosing SCK in dairy cows. Animals enrolled in this study were tested within the transition period, because of their greater risk for developing SCK compared with mid- and late-lactating cows. Further objectives were to compare different hand-held devices for analyzing BHBA concentrations in capillary as well as coccygeal blood. To evaluate whether deviations from the reference test were due to the hand-held devices or the origin of the samples, capillary as well as coccygeal blood were tested with all 3 devices and with the reference test.

The observed prevalence of SCK (BHBA $\geq 1.2 \mathrm{mmol} / \mathrm{L}$ ) was at the lower range of previously reported prevalence of 16.1 to $55.7 \%$ (Geishauser et al., 1998; McArt et al., 2012; Suthar et al., 2013). Even considering only cows within the first 2 wk of lactation, the prevalence of SCK, based on serum BHBA concentrations of 1.2 and $1.4 \mathrm{mmol} / \mathrm{L}$, was low with 14.8 and $8.1 \%$, respectively. The reason for these low prevalences on this particular farm might be the farmers' awareness for SCK, resulting in continuous improvement and implementation of good herd health-management procedures.

The differences in the total number of incisions needed with each lancet to obtain adequate amounts of 
Table 3. Corresponding thresholds and performance of 3 hand-held meters ${ }^{1}$ to detect subclinical ketosis in capillary and coccygeal blood based on BHBA concentrations in serum of 1.2 and $1.4 \mathrm{mmol} / \mathrm{L}$, respectively, obtained from 49 prepartum ${ }^{2}$ and 191 postpartum $^{3}$ Holstein-Friesian cows predominately taken between 2 wk before and 4 wk after calving

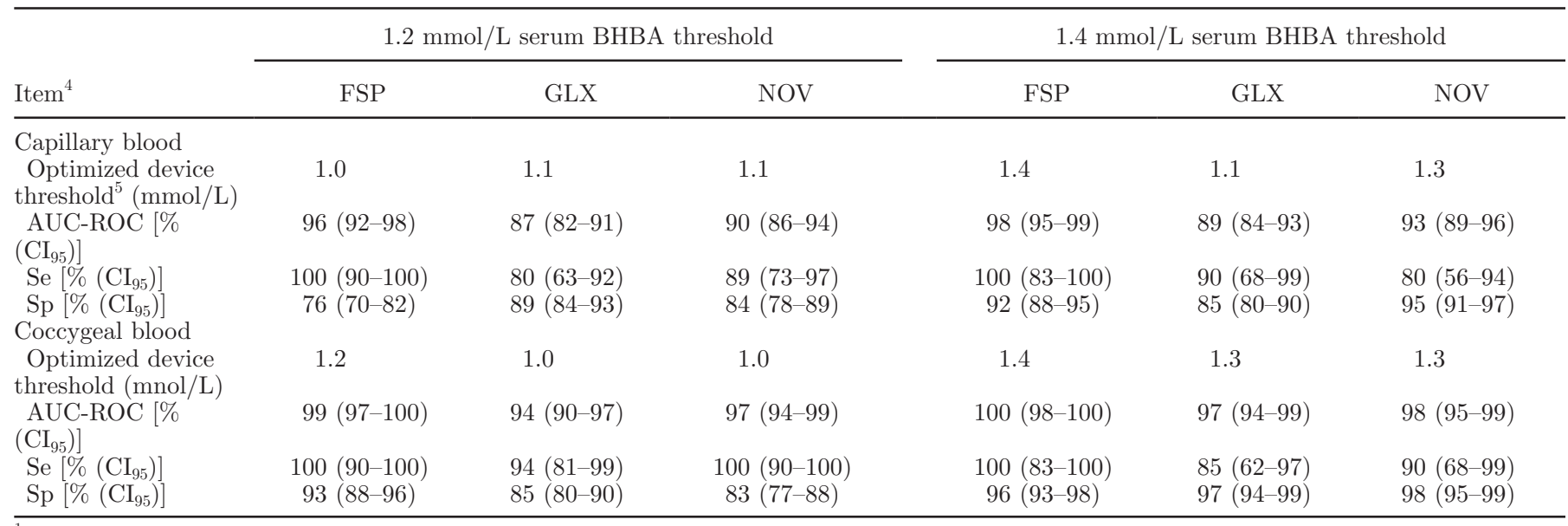

${ }^{1}$ FSP $=$ FreeStyle Precision (Abbott GmbH and Co. KG, Wiesbaden, Germany); GLX = GlucoMen LX Plus (A. Menarini GmbH, Vienna, Austria); NOV = NovaVet (Nova Biomedical, Waltham, MA).

${ }^{2}$ Median: $4 \mathrm{~d}$, minimum: $21 \mathrm{~d}$, maximum: $12 \mathrm{~h}$ prepartum.

${ }^{3}$ Median: $8 \mathrm{~d}$, minimum: $1 \mathrm{~d}$, maximum: $29 \mathrm{~d}$ postpartum.

${ }^{4} \mathrm{AUC}-\mathrm{ROC}=$ area under the receiver operating characteristics $(\mathrm{ROC})$ curve; $\mathrm{CI}_{95}=95 \%$ confidence interval; Se $=$ sensitivity; Sp $=$ specificity

${ }^{5}$ Based on ROC analyses.

blood for analyses are small but statistically significant. The worst result was determined for the lancet with the smallest blade width (SL); hence, sometimes no blood drop could be obtained. Because a minimal amount of incisions is striven for with regard to animal welfare, it is recommended to use lancets with blade widths of at least $1.5 \mathrm{~mm}$. It should be noted that for this study, the blood drop obtained by one incision was used for all 3 hand-held meters, whereas in practice only one device will be used; that is, the amount of blood needed is smaller. Thus, squeezing may be less of a problem in practice than in our study.

All 3 electronic devices used in this study were already evaluated in previous studies (Iwersen et al., 2013; Mahrt et al., 2014b) with blood obtained from a tail vessel as test substrate. For the FSP, Iwersen et al. (2013) determined a $\rho=0.94$ and a mean \pm SD difference of $+0.04 \pm 0.15 \mathrm{mmol} / \mathrm{L}$, and for the GLX device, a $\rho=0.80$ and a mean \pm SD difference of $-0.12 \pm 0.22$, respectively, compared with the laboratory results. For the NOV, Mahrt et al. (2014b) reported a $\rho=0.87$ and a mean $\pm \mathrm{SD}$ difference of $-0.07 \pm 0.17$. Considering coccygeal blood, the determined correlation coefficients and deviations were similar to those already reported. This indicates a good consistency in measurements of the electronic hand-held devices with varying farm conditions as prevalent in the different studies. The main intention of this study, however, was to test whether capillary blood was suitable for diagnosing SCK using electronic hand-held devices.
For the comparison with other studies, rank correlation coefficients were calculated. Kendall's tau $(\tau)$ is an eligible test that is less sensitive to error and discrepancies in the data sets, as well as being easier to interpret than Spearman's rho $(\rho)$. Spearman's rho correlation coefficients are usually larger than $\tau$; hence, a direct comparison of these values is difficult, although the interpretations of both coefficients are often very similar. Because most of the published studies used $\rho$ (Voyvoda and Erdogan, 2010; Iwersen et al., 2013; Mahrt et al., 2014b), the discussion refers to this parameter.

Based on the classification by Taylor (1990), the determined $\rho$ for BHBA concentrations in coccygeal blood determined with the reference test and the handheld devices were regarded as very strong $(\rho \geq 0.9)$ for the FSP and strong ( $\rho$ between 0.68 and 1.0) for the NOV and GLX. The $\rho$ for capillary blood were approximately 12 percentage points lower for the FSP and the NOV, and 18 percentage points lower for the GLX, but still indicating a strong (FSP and NOV) or moderate ( $\rho$ between 0.36 to 0.67 for the GLX) association. The differences in the correlation coefficients may not only be caused by the type of blood sample but might also be influenced by the sampling procedure. According to the manufacturer's manuals, it is important to avoid hemolysis of the blood sample because of its negative effect on the accuracy of the results. While obtaining capillary blood, it was sometimes necessary to squeeze the skin of the vulva to get an adequate amount of blood. According to the Clinical Laboratory Standards 

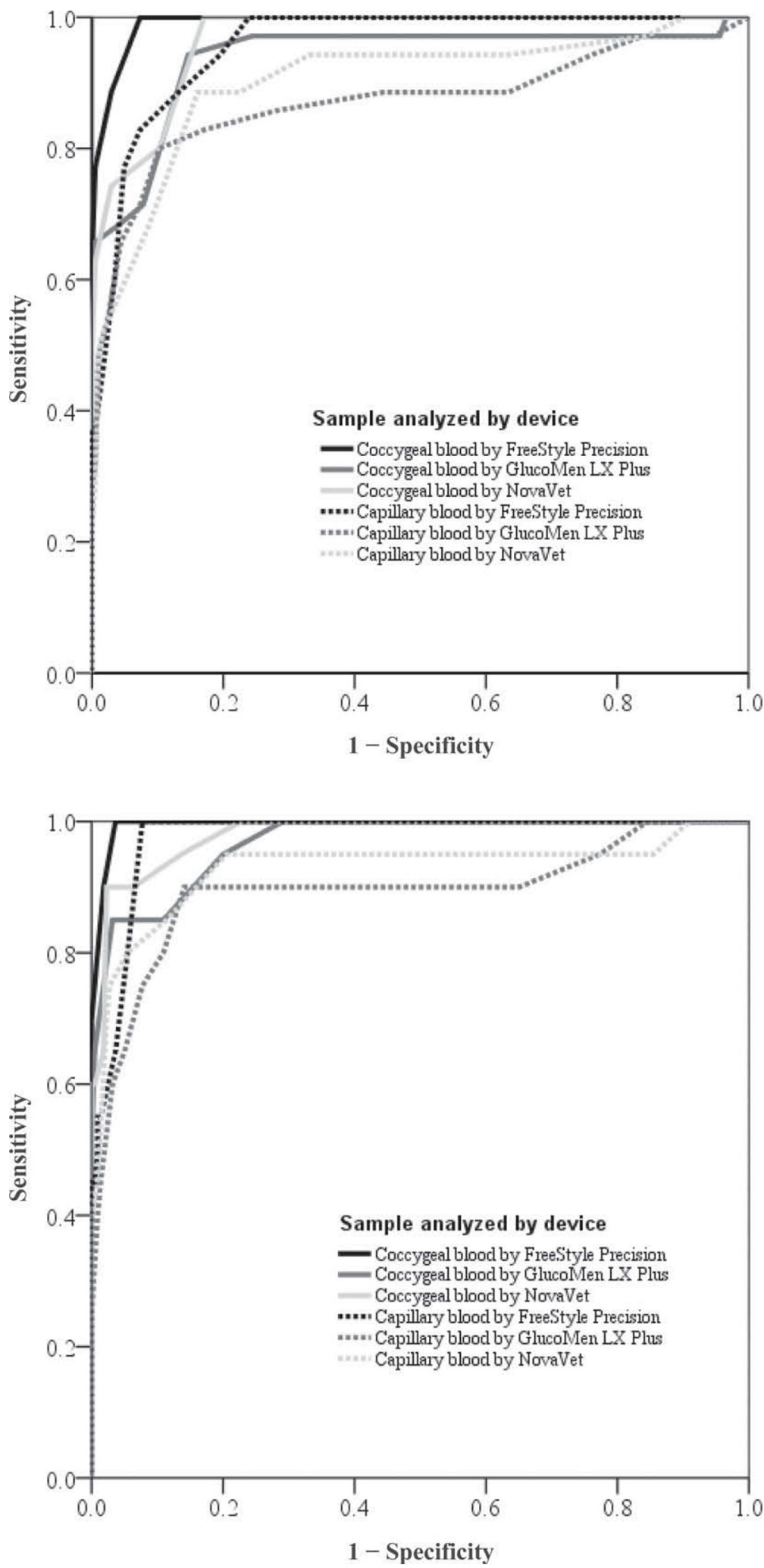

Figure 3. Analyses of receiver operating characteristics for 3 different hand-held devices for diagnosis of ketosis in either coccygeal or capillary blood, using serum BHBA concentrations of $1.2 \mathrm{mmol} / \mathrm{L}$ (top) or $1.4 \mathrm{mmol} / \mathrm{L}$ (bottom) as the threshold for subclinical ketosis. Blood samples were obtained from 49 prepartum and 191 postpartum Holstein-Friesian cows and were predominately taken between 2 wk before and 4 wk after calving. FreeStyle Precision (Abbott GmbH and Co. KG, Wiesbaden, Germany); GlucoMen LX Plus (A. Menarini GmbH, Vienna, Austria); NovaVet (Nova Biomedical, Waltham, MA). 
Institute (CLSI, 2008), squeezing of the skin might increase the risk of hemolysis in capillary blood and could explain the poorer performance. Hence, further research is needed to evaluate the association between the level of squeezing and the BHBA concentration obtained in capillary blood using hand-held devices.

With capillary blood as test substrate, the FSP device overestimated the laboratory results, whereas the GLX and NOV tended to underestimate the BHBA concentrations. From a clinical point of view with regard to the number of mistreated or not-treated animals, these mean variations were acceptable, especially with BHBA concentrations in serum of 1.2 and $1.4 \mathrm{mmol} / \mathrm{L}$, respectively, commonly used as thresholds for SCK.

Plotting the data as presented in Figure 2, heteroscedasticity was observed. The discrepancy between the results of the devices and the laboratory increased with greater BHBA concentrations. This should be considered when interpreting the results, for example, when interpreting the mean BHBA concentrations of the tested devices in capillary and coccygeal blood (Table 1). Therefore, a nonparametric Passing-Bablok regression analyses was performed and revealed a systematic and a proportional difference for almost all devices with both sample types (capillary and coccygeal blood). Hence, ROC analyses were performed to calculate optimized thresholds for the detection of SCK in coccygeal and capillary blood using the electronic hand-held devices. Applying the optimized thresholds, all devices were capable to detect SCK in capillary and coccygeal blood. The observed Se and Sp were within the range of those studies conducted by Iwersen et al. (2013) and Mahrt et al. (2014b), allowing a reliable monitoring of SCK in dairy cows. Additionally, the AUC-ROC reveals good performance for all devices to detect cows suffering from SCK, but results improved numerically when a serum threshold of $1.4 \mathrm{mmol} / \mathrm{L}$ was used and coccygeal blood was used as sample material (Figure 3). Except for the GLX using a BHBA cut-off in serum of $1.4 \mathrm{mmol} / \mathrm{L}$, confidence intervals of calculated AUC-ROC overlapped, indicating no statistical difference between the origin of the sample. The AUC-ROC values classified as excellent were obtained for FSP and NOV, whereas good AUC-ROC values were found for the GLX. Hence, the first 2 mentioned devices were most eligible for detecting SCK in capillary blood.

To evaluate the consistency of the test results, intra- and interassay CV were calculated. The average intraassay CV for the FSP (7.9\%), the GLX (8.3\%), and the NOV device (10.9\%) were below $15 \%$ as requested by the European Medicines Agency (EMEA, 2011) and, hence, in an acceptable range. In general, the intra- and interassay CV improved with greater BHBA concentrations.
The BHBA concentrations in blood are influenced by several factors, such as type of fodder, feeding technique, and diurnal variation (Eicher et al., 1999; Nielsen et al., 2003; Mahrt et al., 2014a). A potential weakness of this study is the composition of the sample set, which was composed of samples collected from animals of one commercial farm with low prevalence of SCK. Further studies should include more farms with varying environmental conditions and prevalence of SCK to prove the external validity of the study results. Additionally, as on-farm tests requiring only a small amount of blood get more and more popular as diagnostic tools in veterinary practice, further knowledge is needed on the concentration of metabolites in capillary blood compared with venous or arterial blood concentrations. In this context, further studies are planned to evaluate the effect of squeezing the skin while collecting a blood drop on the analyzed BHBA concentrations and to further optimize the sampling procedures.

\section{CONCLUSIONS}

The results of this study demonstrate that capillary blood obtained by a minimally invasive technique was eligible to detect SCK using electronic hand-held devices. Even if the observed test characteristics were lower compared with coccygeal blood as test substrate, the results were still in a good range for an on-site test. Hence, this procedure could be recommended, especially for countries where farmers are not allowed to collect conventional blood samples. Based on AUC-ROC analyses, the FSP and NOV showed comparable results and were most eligible in detecting SCK in capillary blood. To avoid an unnecessary amount of punctures, lancets with blade widths of at least $1.5 \mathrm{~mm}$ should be used. Further studies on additional farms are needed to prove the external validity of the study results.

\section{ACKNOWLEDGMENTS}

This study was supported, in part, by a scholarship from the Austrian Association for Buiatrics (Vienna, Austria) and by a grant from Elanco Animal Health (Vienna, Austria). We thank the staff from FirstFarms Slovakia, as well as Alexander Erlen, Armin Hofer, Markus Suda, and David Süss (all from University of Veterinary Medicine Vienna, Vienna, Austria) for their technical assistance.

\section{REFERENCES}

Andersson, L. 1988. Subclinical ketosis in dairy cows. Vet. Clin. North Am. Food Anim. Pract. 4:233-251.

Bilić-Zulle, L. 2011. Comparison of methods: Passing and Bablok regression. Biochem. Med. (Zagreb) 21:49-52. 
Bland, J. M., and D. G. Altman. 1986. Statistical methods for assessing agreement between two methods of clinical measurement. Lancet 1:307-310.

Carrier, J., S. Stewart, S. Godden, J. Fetrow, and P. Rapnicki. 2004. Evaluation and use of three cowside tests for detection of subclinical ketosis in early postpartum cows. J. Dairy Sci. 87:3725-3735.

Chapinal, N., M. Carson, T. F. Duffield, M. Capel, S. Godden, M. Overton, J. E. P. Santos, and S. J. LeBlanc. 2011. The association of serum metabolites with clinical disease during the transition period. J. Dairy Sci. 94:4897-4903.

Chapinal, N., S. J. LeBlanc, M. E. Carson, K. E. Leslie, S. Godden, M. Capel, J. E. P. Santos, M. W. Overton, and T. F. Duffield. 2012. Herd-level association of serum metabolites in the transition period with disease, milk production, and early lactation reproductive performance. J. Dairy Sci. 95:5676-5682.

CLSI. 2008. Procedures and Devices for the Collection of Diagnostic Blood Specimens by Skin Puncture; Approved Standard. 6th ed. Clin. Lab. Stand. Inst., Wayne, PA.

Cook, N., G. Oetzel, and K. Nordlund. 2006. Modern techniques for monitoring high-producing dairy cows - 1 . Principles of herd-level diagnoses. In Pract. 28:510-515.

Dohoo, I. R., and S. W. Martin. 1984. Subclinical ketosis: Prevalence and associations with production and disease. Can. J. Comp. Med. 48:1-5.

Duffield, T. F., D. F. Kelton, K. E. Leslie, K. D. Lissemore, and J. H. Lumsden. 1997. Use of test day milk fat and milk protein to detect subclinical ketosis in dairy cattle in Ontario. Can. Vet. J. $38: 713-718$.

Duffield, T. F., K. D. Lissemore, B. W. McBride, and K. E. Leslie 2009. Impact of hyperketonemia in early lactation dairy cows on health and production. J. Dairy Sci. 92:571-580.

Duffield, T. F., D. Sandals, K. E. Leslie, K. Lissemore, B. W. McBride, J. H. Lumsden, P. Dick, and R. Bagg. 1998. Efficacy of monensin for the prevention of subclinical ketosis in lactating dairy cows. J. Dairy Sci. 81:2866-2873.

Eicher, R., A. Liesegang, E. Bouchard, and A. Tremblay. 1999. Effect of cow-specific factors and feeding frequency of concentrate on diurnal variations of blood metabolites in dairy cows. Am. J. Vet. Res. 60:1493-1499.

EMEA (European Medicines Agency). 2011. Guideline on bioanalytical method validation. Accessed May 3, 2015. http://www. ema.europa.eu/docs/en_GB/document_library/Scientific_guideline/2011/08/WC500109686.pdf.

Geishauser, T., K. Leslie, D. Kelton, and T. Duffield. 1998. Evaluation of five cowside tests for use with milk to detect subclinical ketosis in dairy cows. J. Dairy Sci. 81:438-443.

Geishauser, T., K. Leslie, J. Tenhag, and A. Bashiri. 2000. Evaluation of eight cow-side ketone tests in milk for detection of subclinical ketosis in dairy cows. J. Dairy Sci. 83:296-299.

Iwersen, M., U. Falkenberg, R. Voigtsberger, D. Forderung, and W. Heuwieser. 2009. Evaluation of an electronic cowside test to detect subclinical ketosis in dairy cows. J. Dairy Sci. 92:2618-2624.

Iwersen, M., D. Klein-Jöbstl, M. Pichler, L. Roland, B. Fidlschuster, I. Schwendenwein, and M. Drillich. 2013. Comparison of 2 electronic cowside tests to detect subclinical ketosis in dairy cows and the influence of the temperature and type of blood sample on the test results. J. Dairy Sci. 96:7719-7730.
Krogh, M. A., N. Toft, and C. Enevoldsen. 2011. Latent class evaluation of a milk test, a urine test, and the fat-to-protein percentage ratio in milk to diagnose ketosis in dairy cows. J. Dairy Sci. 94:2360-2367.

Mahrt, A., O. Burfeind, and W. Heuwieser. 2014a. Effects of time and sampling location on concentrations of $\beta$-hydroxybutyric acid in dairy cows. J. Dairy Sci. 97:291-298.

Mahrt, A., O. Burfeind, R. Voigtsberger, A. Müller, and W. Heuwieser. 2014b. Evaluation of a new electronic handheld meter for measurement of $\beta$-hydroxybutyric acid in dairy cows. Tierarztl. Prax. Ausg. G Grosstiere Nutztiere 42:5-10.

McArt, J. A. A., D. V. Nydam, and G. R. Oetzel. 2012. A field trial on the effect of propylene glycol on displaced abomasum, removal from herd, and reproduction in fresh cows diagnosed with subclinical ketosis. J. Dairy Sci. 95:2505-2512.

Nielsen, N. I., K. L. Ingvartsen, and T. Larsen. 2003. Diurnal variation and the effect of feed restriction on plasma and milk metabolites in TMR-fed dairy cows. J. Vet. Med. A Physiol. Pathol. Clin. Med. 50:88-97.

Passing, H. and Bablok. 1983. A new biometrical procedure for testing the equality of measurements from two different analytical methods. Application of linear regression procedures for method comparison studies in clinical chemistry, Part I. J. Clin. Chem. Clin. Biochem. 21:709-720.

Pichler, M., A. Damberger, I. Schwendenwein, J. Gasteiner, M. Drillich, and M. Iwersen. 2014. Thresholds of whole-blood $\beta$-hydroxybutyrate and glucose concentrations measured with an electronic hand-held device to identify ovine hyperketonemia. J. Dairy Sci. 97:1388-1399.

Roberts, T., N. Chapinal, S. J. LeBlanc, D. F. Kelton, J. Dubuc, and T. F. Duffield. 2012. Metabolic parameters in transition cows as indicators for early-lactation culling risk. J. Dairy Sci. 95:30573063.

Rollin, E., R. D. Berghaus, P. Rapnicki, S. M. Godden, and M. W. Overton. 2010. The effect of injectable butaphosphan and cyanocobalamin on postpartum serum $\beta$-hydroxybutyrate, calcium, and phosphorus concentrations in dairy cattle. J. Dairy Sci. 93:978 987.

Suthar, V. S., J. Canelas-Raposo, A. Deniz, and W. Heuwieser. 2013 Prevalence of subclinical ketosis and relationships with postpartum diseases in European dairy cows. J. Dairy Sci. 96:2925-2938.

Swets, J. A. 1988. Measuring the accuracy of diagnostic systems. Science 240:1285-1293.

Taylor, R. 1990. Interpretation of the correlation coefficient: A basic review. J. Diagn. Med. Sonogr. 6:35-39.

Voyvoda, H., and H. Erdogan. 2010. Use of a hand-held meter for detecting subclinical ketosis in dairy cows. Res. Vet. Sci. 89:344351.

Walsh, R. B., D. F. Kelton, T. F. Duffield, K. E. Leslie, J. S. Walton, and S. J. LeBlanc. 2007a. Prevalence and risk factors for postpartum anovulatory condition in dairy cows. J. Dairy Sci 90:315-324.

Walsh, R. B., J. S. Walton, D. F. Kelton, S. J. LeBlanc, K. E. Leslie, and T. F. Duffield. 2007b. The effect of subclinical ketosis in early lactation on reproductive performance of postpartum dairy cows. J. Dairy Sci. 90:2788-2796. 